\title{
Polysèmes
}

Revue d'études intertextuelles et intermédiales

\section{Le texte et le tableau comme sas intertextuels dans Midnight's Children de S. Rushdie et Ever After de G. Swift}

\section{Catherine Pesso-Miquel}

\section{(2) OpenEdition}

Journals

Édition électronique

URL : http://journals.openedition.org/polysemes/1610

DOI : 10.4000/polysemes. 1610

ISSN : 2496-4212

Éditeur

SAIT

Édition imprimée

Date de publication : 1 janvier 2003

Pagination : 95-125

ISSN : 0999-4203

\section{Référence électronique}

Catherine Pesso-Miquel, « Le texte et le tableau comme sas intertextuels dans Midnight's Children de S. Rushdie et Ever After de G. Swift », Polysèmes [En ligne], 6 | 2003, mis en ligne le 10 janvier 2017, consulté le 20 avril 2019. URL : http://journals.openedition.org/polysemes/1610 ; DOI : 10.4000/ polysemes. 1610

Ce document a été généré automatiquement le 20 avril 2019.

Polysèmes 


\title{
Le texte et le tableau comme sas intertextuels dans Midnight's Children de S. Rushdie et Ever After de G. Swift
}

\author{
Catherine Pesso-Miquel
}

1 Un sas, dit le dictionnaire, est entre autres choses « une petite pièce étanche entre deux milieux différents (air et eau, air et absence d'air) qui permet le passage ». Cette modalité particulière du passage, banale à l'intérieur des sous-marins ou des engins spatiaux, me paraît un modèle utile pour rendre compte de certains phénomènes, à la fois intertextuels et interculturels, à l'œuvre dans deux romans britanniques contemporains, Midnight's Children de Salman Rushdie et Ever After de Graham Swift. Nous examinerons des cas d'intertextualité médiatisée dans lesquels une œuvre, ou un élément d'une œuvre, sert de « sas ", qui permet à deux cultures distinctes d'entrer en contact, ou qui sert de transition entre deux autres œuvres.

2 Cette intertextualité particulière ouvre des brèches dans les parois étanches qui séparent les identités nationales ou les époques historiques, et elle problématise les concepts de «filiation» littéraire et de parodie. Que produit la mise en contact, au moyen de ces « sas », de deux cultures : conflit, effacement de l'une par l'autre, mélange heureux ? Et quelle est la fonction du «sas» si l'on s'en tient à une relation strictement intertextuelle? Genette utilise la métaphore du palimpseste pour mieux définir l'intertextualité, mais dans son livre Palimpsestes il réfléchit essentiellement sur des relations binaires, entre deux textes, qu'il appelle hypotexte et hypertexte. Mais que se passe-t-il lorsqu'un troisième texte vient se superposer, sur le parchemin, à deux textes imparfaitement effacés? Quelles relations le troisième texte (hyper-hypertexte ?) peut-il entretenir avec l'hypotexte d'une part, avec l'hypertexte d'autre part? Si le texte deux, ou hypertexte, est déjà une parodie, ou comme dirait Umberto Eco, «an ironic rethinking $»^{1}$ du déjà dit, le troisième texte n'est-il alors qu'une surenchère, un degré de 
plus dans la «re-visite » du passé, dans la déformation comique ? Tout en réfléchissant à ces questions, nous tenterons de voir comment ce type particulier d'intertextualité s'inscrit dans le paysage littéraire contemporain en général, et comment il s'inscrit dans le champ - aux limites floues et souvent contestées - du roman postmoderniste.

\section{Tableau et horloge : sas interculturels chez Rushdie}

3 Le tableau de Millais intitulé The Boyhood of Raleigh, explicitement cité dans Ever After, fait aussi l'objet d'allusions constantes de la part de Saleem Sinai, le narrateur de Midnight's Children. Sir John Everett Millais est surtout connu en tant que peintre préraphaélite, mais The Boyhood of Raleigh date de l'époque où il avait abandonné les techniques, qu'il jugeait trop longues et trop coûteuses, du mouvement qu'il avait fondé avec Rossetti et Hunt, pour apporter sa contribution à cette production picturale conventionnelle et moralisante connue sous l'étiquette de "peinture victorienne ». L'œuvre, peinte in situ, sur la côte du Devonshire, fut décrite en ces termes par Stevens, un critique d'art de l'époque :

This work glows in the warm light of the Devonshire sun, and shows the sunburnt, stalwart Genoese sailor-one of those who were half pirates, half heroes, such as Kingsley has delighted countless boys by describing-seated, with his brawny, bronzed shoulders towards us, on a sea wall, while before him, and at ease upon the floor, are Raleigh and his brother, listening eagerly and with rapt ears to the narration of wonders on sea and land. ${ }^{2}$

Le tableau date de 1871, et la reine Victoria fut couronnée Impératrice des Indes cinq ans plus tard, en 1876. The Boyhood of Raleigh s'inscrit donc dans l'apologie triomphante de l'impérialisme qui prévalait en cette fin du dix-neuvième siècle; ce tableau occulte les revers essuyés par les expéditions de Raleigh, ainsi que sa mort sur l'échafaud, pour faire de lui un enfant appelé au glorieux destin de constructeur de l'Empire britannique. Il n'y a donc rien d'étonnant à ce que ce tableau ait été maintes fois reproduit, et soit devenu pour les Anglais une sorte d'image d'Epinal, fréquemment choisie pour décorer une chambre de garçon, ou illustrer des livres d'aventures. D'autre part, l'iconographie du tableau ne renvoie pas du tout à une représentation historique réaliste de l'époque Tudor: les enfants (dont l'un deviendra Sir Walter, mais qui pour l'instant n'est qu'un roturier) sont représentés comme de petits princes, vêtus de costumes de velours; ils correspondent à une vision idéalisée, victorienne, d'une enfance docile, propre, bridée, et semblent anticiper Little Lord Fauntleroy, dont le costume de velours allait bientôt devenir célèbre et lancer une mode. Ainsi la scène peinte par Millais est déjà en soi une 
zone trouble, ambiguë, un mélange chimérique et impossible de deux époques et de deux cultures.

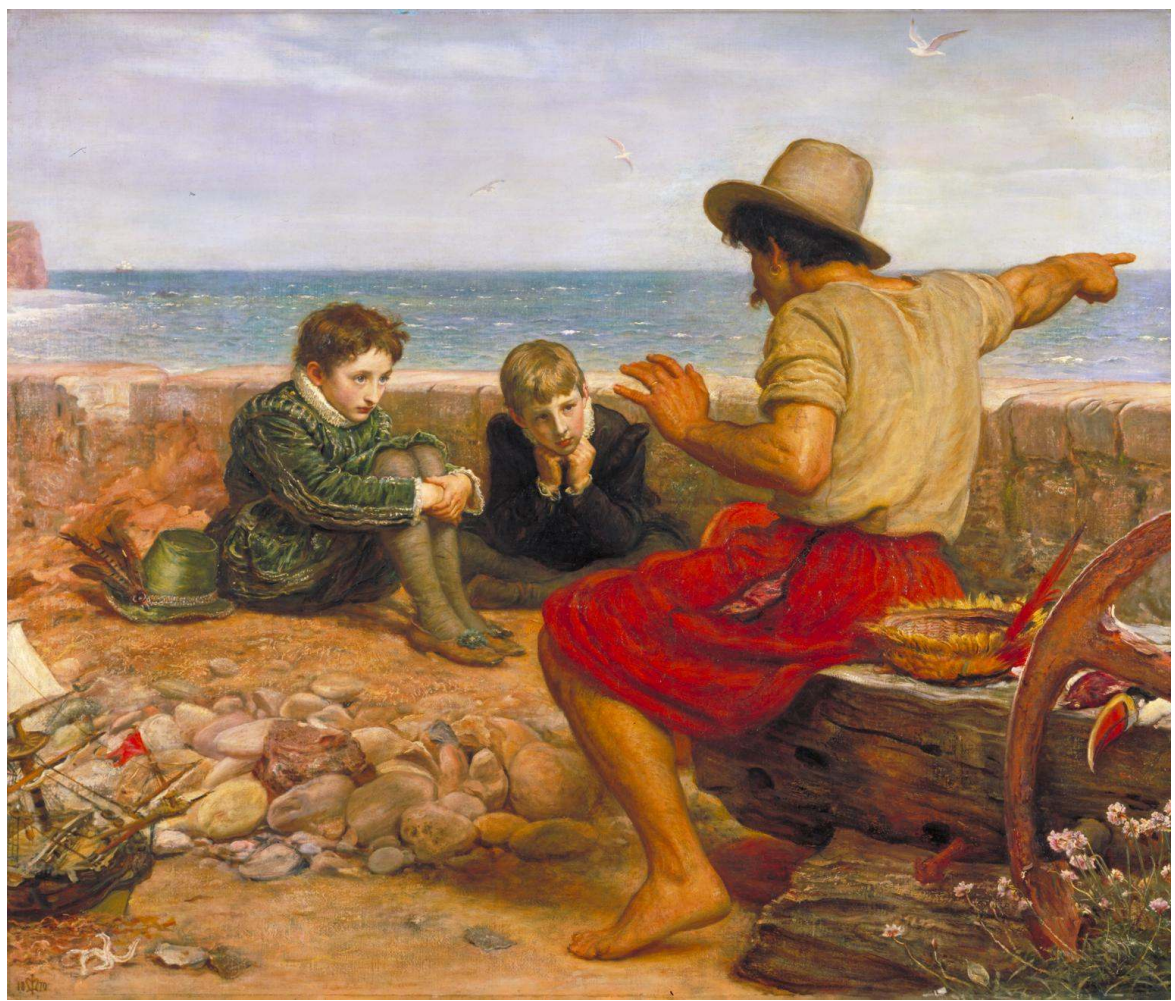

John Everett Millais, The Boyhood of Raleigh (1871), huile sur toile, 1206 x 1422 mm, Tate Collection Creative Commons

Dans Midnight's Children, les parents du narrateur achètent à l'Anglais Methwold l'une des maisons d'un lotissement, autrefois louées à des Anglais partis précipitamment à l'approche de l'indépendance. Mais si Methwold en demande un prix ridiculement bas, il $\mathrm{y}$ met une condition expresse et très symbolique : ce n'est que le 15 août 1947 à zéro heures (ou, plus poétiquement, à minuit, heure $\mathrm{H}$ de l'indépendance de l'Inde, l'heure très importante sur laquelle le roman s'ouvre, et qui en fournit le titre) que les acheteurs indiens pourront se débarrasser du mobilier et des objets divers et variés laissés sur place par les anciens locataires. Une reproduction de notre tableau, dûment encadrée, figure parmi ce bric-à-brac, témoignant d'ailleurs par sa présence du fait que, juste avant l'abandon honteux et hâtif de leur ancienne colonie, les Anglais ressentaient une nostalgie certaine, et s'accrochaient à leurs rêves ancestraux de règne, de puissance, de gloire... et de richesse. Or cette reproduction encadrée du tableau de Millais fait justement partie des très rares objets que les Sinai gardent chez eux après l'heure fondatrice et fatidique - «the picture on my wall survived ", nous dit Saleem (MC 128) et cette image que le narrateur aura gardée sous les yeux tout au long de son enfance nourrit son imagination, et reste présente dans sa mémoire.

En effet le doigt pointé qui sert de point de fuite au tableau prête son titre au premier chapitre de la deuxième partie du roman («The fisherman's pointing finger »), et donne naissance à un jeu métaphorique complexe où les doigts reviennent en un leitmotiv lancinant : le doigt coupé de Saleem, le doigt pointé du minaret, le doigt accusateur du rhésus, "pointing its unanswerable finger» (MC 236). L'image est doublement utile: d'une part elle aide Saleem à se représenter son aïeul Aadam Aziz alors qu'il était encore 
un petit garçon fasciné par les récits d'un vieux batelier : «Tai, forecasting the fisherman on my wall, pointed at the mountain » (MC 16), entrant ainsi dans la création paranoïaque d'une toile d'araignée où se croisent et s'entrecroisent analogies, parallèles et répétitions. C'est un jeu auquel se livrent, avec la même passion, et Swift et Rushdie. D'autre part, ce petit Raleigh aux yeux émerveillés, suspendu aux lèvres du marin, offre à Rushdie une métaphore auto-réflexive implicite lui permettant de mettre en abyme son propre roman, qui est en fait un hymne à l'oralité dans lequel un conteur serpent hypnotise les mangoustes que sont Padma et le lecteur.

7 Jamais Saleem ne mentionne précisément le nom du peintre ou le titre exact du tableau, mais on trouve dans Midnight's Children plus de trente références, directes ou indirectes, à ce tableau suspendu dans la chambre de Saleem, représentant «the boy Raleigh », que le lecteur identifie grâce à la description qui en est faite. Pourtant le narrateur le décrit de mémoire, alors qu'il est devenu adulte, et que la reproduction - et la villa elle-même - ont disparu depuis longtemps. Cela explique pourquoi le marin qu'il dépeint ne correspond pas tout à fait au personnage créé par Millais : « an old, gnarled, net-mending sailor-did he have a walrus moustache? ? (MC 122). Le marin du tableau a bien une moustache de morse, mais il semble jeune, ne répare aucun filet, et, avec sa boucle d'oreille, ressemble plutôt à l'un des hardis corsaires de Drake, qui ne s'embarrassaient pas de scrupules quand ils abordaient un vaisseau arborant le pavillon espagnol.

8 Jouant sur la figure anonyme du deuxième petit garçon (que le critique Stevens identifie comme étant le frère de Raleigh), Saleem demande :

The young Raleigh-and who else ? - sat, framed in teak [...] Raleigh-and who

else?

Because there was certainly another boy in the picture, sitting cross-legged in frilly

collar and button-down tunic... (MC 122)

Or cet enfant va descendre du mur pour envahir l'identité de Saleem, qui se voit contraint d'endosser pour son septième anniversaire une copie, exécutée par un tailleur, du costume que l'enfant porte dans le tableau de Millais. Affublé de ce costume, « hot and constricted in the outlandish garb» (MC 156), Saleem suscite l'attendrissement de sa mère et de ses voisines :

"Look, how chweet!" Lila Sabarmati exclaimed to my eternal mortification, "It's like

he's just stepped out of the picture!" (MC 122)

"So chweet!" [Amina cried] (MC 156)

10 Avec l'expression «stepped out of the picture », l'univers anglais déborde effectivement du tableau pour envahir et annexer l'espace indien: les limites qui devraient séparer des univers distincts deviennent brusquement perméables.

11 Rushdie a affirmés qu'il avait cherché, en écrivant ce roman, à se démarquer du roman anglo-indien en général et du Raj Quartet de Paul Scott en particulier. Le premier roman de ce quatuor, The Jewels in the Crown, emprunte son titre à celui d'un tableau, suspendu au mur d'une salle de classe, qui représente la reine Victoria assise sur son trône, entourée de ses ministres et dignitaires, et recevant l'hommage d'un prince indien. Le narrateur imaginé par Scott insiste sur la fausseté de cette représentation, filtrée par les fantasmes des colonisateurs: "The India of the picture had never existed outside its gilt frame ${ }^{4}$ ». Ironiquement, le personnage de l'institutrice, Miss Crane, se sert de cette Inde irréelle, bien contenue, pour enseigner le vocabulaire anglais aux petits Indiens.

$12 \mathrm{Au}$ contraire, avec l'épisode du déguisement, Rushdie ouvre le sas du tableau; le cadre étanche peut alors laisser le passage et permettre à un univers anglais tout aussi fictif que 
l'Inde évoquée par Scott (l'Angleterre Tudor de l'enfance de Raleigh revue et corrigée par un victorien) de pénétrer l'univers indien. La transformation de Saleem en petit noble vêtu de velours provoque chez sa mère et les amies de celle-ci un accès de cette sentimentalité sirupeuse si fréquente chez les lecteurs des romans de Frances Hodgson Burnett ${ }^{5}$. Dans ce sens, la « colonisation » symbolique n'a rien d'étonnant, et ailleurs dans le roman Saleem dénonce vigoureusement la fascination exercée sur les classes dirigeantes indiennes par le modèle anglais.

13 Fait plus surprenant, qui nous permet de filer la métaphore du sas, Rushdie inverse le sens de la « colonisation », car Saleem pénètre à l'intérieur du tableau, il s'engouffre à son tour dans le sas pour envahir l'espace anglais, en occupant l'identité restée vacante de l'enfant anonyme : «In a picture hanging on a bedroom wall, I sat beside Walter Raleigh and followed a fisherman's pointing finger with my eyes " (MC 122, je souligne). Ce phénomène d'interpénétration se produit d'ailleurs dès la première description du tableau:

Memory of my blue bedroom wall: on which, next to the P.M.'s letter, the Boy Raleigh hung for many years, gazing rapturously at an old fisherman in what looked like a red dhoti, who sat on-what?-driftwood?-and pointed out to sea as he told his fishy tales... (MC 15)

14 En effet, ici, l'imagination de Saleem habille le personnage de Millais d'un vêtement indien ; sa culotte rouge devient un « dhoti » indigène, et la transformation du corsaire en pêcheur est une stratégie délibérée chez Rushdie, appuyée par l'emploi des mots " fisherman » et "fishy ». Le personnage de Millais, ainsi « recyclé », s'intégre dans un réseau métaphorique complexe, propre à Midnight's Children, où pêcheurs et poissons jouent un rôle important. Son doigt pointé, nous dit le narrateur, indique, au-delà du cadre, les voiles rouges des bateaux de pêcheurs Koli, comme si le drapeau rouge à la proue du voilier-jouet peint dans le coin inférieur gauche était sorti du tableau pour s'intégrer au paysage, adopter la couleur locale, et devenir un « dhow » Koli :

[I]f one followed [the fisherman's pointing finger] even further, it led one out through the window, [...] out to another sea which was not the sea in the picture; a sea on which the sails of Koli dhows glowed scarlet in the setting sun... an accusing finger, then, which obliged us to look at the city's dispossessed. (MC 123)

Il y a donc vraiment circulation dans les deux sens, le tableau de Millais devient une zone à la fois étanche et perméable, ou un sas, par lequel les deux univers entrent en contact et « coulent l'un dans l'autre » ce qui constitue une subtile subversion d'une œuvre picturale qui véhicule l'impérialisme victorien. La colonisation de l'Inde par l'Angleterre côtoie une « colonisation » de l'Angleterre par l'Inde qui se rit de la chronologie, du réalisme et de la vraisemblance. On a ici un exemple de recentrage culturel: Rushdie rappelle narquoisement à ses lecteurs que la culture indienne existait bien avant que les Européens ne s'avisent de "découvrir l'Inde ». Cependant, au-delà d'une rivalité culturelle, il convient de voir là un phénomène d'hybridation: l'Inde et l'Angleterre se fondent l'une dans l'autre comme les saveurs, comme les humains :

Perhaps we are all, black and brown and white, leaking into one another, as a character of mine once said, like flavours when you cook. ${ }^{6}$

La façon dont le pronom «I " envahit l'espace du tableau et se coule dans une identité restée vacante souligne subtilement l'une des angoisses de Saleem, liée à son identité instable et changeante. Comme Oskar, le narrateur du Tambour de Günter Grass, Saleem ne sait ni qui il est, ni ce qu'il est, et la narration à la première personne se fait hésitante, annexe souvent la troisième personne. À l'intérieur d'une même phrase, et avec une 
rapidité déconcertante, l'Oskar de Grass est capable de passer plusieurs fois de suite de la première à la troisième personne, du pronom Ich au prénom Oskar, toujours pour se désigner lui-même. Saleem fait de même à plusieurs reprises, par exemple dans le chapitre intitulé «In the Sundarbans ». On voit ici à l'œuvre une des différences entre romans modernistes et romans postmodernistes : selon Brian McHale, les premiers ont une dominante épistémologique, et les seconds une dominante ontologique. Effectivement, les romans modernistes sont centrés sur des sujets, dont la conscience est rendue transparente par l'écriture d'un monologue intérieur, et que le lecteur doit tenter de connaître, d'appréhender dans leur globalité et leur complexité, alors que dans les romans postmodernistes le sujet est tellement instable, fluctuant et fragmentaire, qu'il est impossible de le définir et de le connaître, que toute approche épistémologique devient vaine.

Rushdie exploite le doigt pointé du marin de Millais pour le "récupérer » et l'intégrer dans son roman, une œuvre où les rapports analogiques s'entrecroisent et où les métaphores sont inoriginées comme dirait Barthes, c'est-à-dire qu'elles sont " des chaînes de substitutions dans lesquelles on s'abstient de repérer un terme premier, fondateur ${ }^{7}$. En effet, si d'après Saleem l'index pointé du prétendu pêcheur désigne, au-delà du cadre du tableau, la baie de Bombay, avec ses dhows et ses poissons, les poissons de la baie, eux, se transforment à leur tour en doigts pointés de mauvais augure :

Chowpatty Beach, and Juhu and Trombay, too were littered with the ominous corpses of dead pomfret, which floated, without the ghost of an explanation, bellyside-up, like scaly fingers in to shore. ${ }^{8}$

Il est un deuxième exemple, dans Midnight's Children, de mélange des cultures indienne et anglaise, mais c'est une horloge, cette fois-ci, qui fait office de sas, et qui met en contact l'Orient et l'Angleterre, en une allusion transparente à l'un des hypotextes du roman, qui est le Tristram Shandy de Sterne. Saleem Sinai, comme Tristram Shandy, se lamente sur le temps qu'il lui faut pour raconter sa propre vie, et insiste sur la durée de l'acte d'énonciation du texte, ce qui d'ailleurs le classe dans une catégorie narratologique très rare ${ }^{9}$. Saleem, comme Tristram avant lui, risque de mourir avant d'avoir, comme dirait Padma, « [got him]self born $»^{10}$.

Avant de pouvoir narrer sa naissance, Tristram doit expliquer les circonstances de sa conception. La façon loufoque dont Sterne a parodié pour ce faire les théories de Locke sur l'association d'idées est restée célèbre : dans l'esprit de la mère du héros, les deux actes que son mari accomplit ponctuellement le premier dimanche de chaque mois, à savoir, remonter l'horloge familiale d'une part, et s'acquitter de son devoir conjugal d'autre part, sont inextricablement liés. D'où la fameuse et malencontreuse question adressée par Mme Shandy à son mari au moment délicat de la conception de Tristram, «pray, my dear, have you not forgot to wind up the clock? »" l'allusion à Sterne est transparente. Dès le premier chapitre, Saleem évoque sa naissance miraculeuse, qu'il appelle "my clock-ridden birth». Le mot clock apparaît dès le deuxième paragraphe de l'incipit :

I was born [...] on the stroke of midnight. Clock-hands joined palms in respectful greeting as I came. [...] [T]hanks to the occult tyrannies of those blandly saluting clocks I had been mysteriously handcuffed to history, my destinies indissolubly chained to those of my country. (MC 9) 
21 culture, au moyen de l'allusion au solennel salut oriental, qui consiste en une courbette, exécutée avec les deux paumes jointes devant la poitrine, ce qui redonne vie à la métaphore morte présente dans le nom composé «clock-hands». Les deux aiguilles de l'horloge, jointes à minuit, évoquent un salut oriental presque caricatural, respectueux mais impénétrable et vaguement inquiétant («blandly saluting clocks»), comme si Rushdie s'amusait à reprendre ironiquement à son compte les stéréotypes orientalistes dénoncés par Edward Said dans son étude Orientalism ${ }^{12}$. L'horloge fournit donc à Rushdie une autre occasion d'inverser le sens habituel de l'annexion, de la colonisation culturelle, comme si le sas était une machine à remonter le temps, ou même à synchroniser.

Avec ces jeux sur le tableau de Millais et l'horloge de Sterne, l'intertextualité devient un exemple d'interpénétration et d'enrichissement mutuels de deux cultures. On voit ici à l'œuvre une stratégie délibérée d'hybridation, de fusion harmonieuse et réussie de deux identités nationales et de deux traditions littéraires radicalement différentes, par le biais de sas interculturels constitués par l'allusion à une œuvre antérieure. Rushdie est un partisan convaincu de la «bonne » hybridation, et s'élève contre la nostalgie mal avisée de la pureté identitaire et culturelle, manifeste en Inde chez les fondamentalistes, qu'ils soient hindous ou musulmans.

Rushdie est conscient que se mêlent en lui des influences occidentales et orientales, et même si le personnage de Saleem n'a jamais posé le pied en Angleterre, l'auteur nous rappelle, entre les lignes, que le roman a bel et bien été écrit loin de l'Inde, en Angleterre. Ce n'est pas un hasard si le narrateur se montre souvent en train de rédiger les pages que nous lisons à la lumière d'une lampe "Anglepoise », par exemple lorsqu'il évoque son « nocturnal pool of Anglepoised light» (MC 166) ou son « Anglepoiselit writing ( $(M C$ 38). « Anglepoise » est une marque de lampes de bureau qui se prête à un jeu de mots, car elle évoque irrésistiblement le terme Anglo. Dans la bouche d'Amina Sinai, Anglo est un mot péjoratif, chargé de mépris, qu'elle emploie pour désigner les secrétaires de son mari, aux noms portugais et chrétiens hérités de la colonisation portugaise de Bombay, des noms «bizarres », dit-elle, «Sulaco and Colaco and I don't know what » (MC 133), qu'Amina associe au Coca-Cola, symbole pour elle d'une culture occidentale qu'elle réprouve. Pour Rushdie lui-même, le terme est beaucoup plus ambigu, et le glissement de "Anglepoise » à "Anglo " permet à un éclairage culturel anglais de baigner les feuillets que remplit Saleem. Par le pouvoir de cette lampe ambiguë, le « génie » de l'auteur est convoqué dans son œuvre et vient se superposer à la personne fictive de Saleem. Derrière le narrateur se profile la personne réelle de l'auteur, exilé loin d'un pays perdu, condamné à ne le retrouver qu'à travers les filtres du temps et de la migration, comme il le dit dans son essai « Imaginary Homelands ».

Rushdie prone la pluralité, la bonne hybridation, ce qu'il appelle dans The Satanic Verses l'arbre-chimère miraculeux, qu'il oppose à des exemples de mauvaise hybridation, «the uselessness of mermen, the failures of plastic surgery, the Esperanto-like vacuity of much modern art, the Coca-Colonization of the planet ${ }^{13}$ ». L'apologie de la fusion, implicite dans les sas interculturels de Midnight's Children, est explicite lorsque Rushdie commente The Satanic Verses:

The Satanic Verses celebrates hybridity, impurity, intermingling, the transformation that comes of new and unexpected combinations of human beings, cultures, ideas, politics, movies, songs. It rejoices in mongrelization and fears the absolutism of the Pure. ${ }^{14}$ 
(heureusement pour lui, Rushdie a d'abord offensé, avec Midnight's Children, ceux de ses lecteurs indiens qui étaient farouchement nationalistes, avant d'essuyer le courroux des fondamentalistes musulmans et de tous ceux qui croient que «intermingling with a different culture will inevitably weaken and ruin their own $»^{15}$.

\section{Le troisième texte}

La fameuse horloge de Sterne, dont nous venons de constater l'exploitation par Rushdie, est également présente, en filigrane, dans Ever After, mais le rapport intertextuel entre Sterne et Swift passe par un roman intermédiaire : The French Lieutenant's Woman, de John Fowles.

Dans Ever After, le narrateur, Bill Unwin, professeur de littérature anglaise, écrit un livre sur son aïeul, Matthew Pearce, dont sa mère lui a légué le journal intime. Ce Matthew est un homme du dix-neuvième siècle, un victorien, un contemporain des héros de Fowles. En vacances à Lyme Regis, le jeune Matthew Pearce est profondément choqué lorsqu'il se trouve soudain en présence d'un ichtyosaure, spécimen d'une espèce disparue, et preuve tangible des " erreurs ", et du " gaspillage » dont le Créateur se serait rendu coupable; ce choc cause en lui une crise intellectuelle et une remise en question de sa foi qui seront ensuite aggravées par la parution du livre de Darwin, De l'origine des espèces. Miné par le doute, incapable de vivre dans le mensonge, il doit renoncer à sa femme, fille de pasteur, car son beau-père exige qu'il choisisse entre sa famille et ses croyances impies.

Les liens intertextuels entre Ever After et The French Lieutenant's Woman sont multiples et complexes, et je me contenterai ici de rappeler rapidement que Charles Smithson, le héros de Fowles, est un paléontologue amateur, un adepte des théories de Darwin (que le père de sa fiancée réprouve), qu'il perd sa foi à la fin du roman et que Fowles emprunte la plupart de ses épigraphes à Tennyson, justement le poète sur qui Katherine Potter, personnage d'Ever After, écrit son mémoire.

29 Fiancé à Ernestina, et amoureux de Sarah, Charles Smithson se sent incapable de trahir ses serments, et doit se résoudre à mentir, à feindre d'aimer sa fiancée. Dans le chapitre 44, dans une scène dont la "réalité » se trouve d'ailleurs annulée dans le chapitre suivant (ou mise "sous rature ", comme dirait Brian McHale après Derrida ${ }^{16}$ ), Charles trouve Ernestina occupée à broder une petite pochette destinée à contenir une montre d'homme. Un côté de la petite poche arbore un cœur, frappé des initiales $\mathrm{C}$ et $\mathrm{E}$ (Charles et Ernestina), tandis que sur l'autre côté Ernestina a brodé le début d'une injonction, " "Each time thy watch thou wind" " ${ }^{17}$. Charles émet des hypothèses plaisantes sur la suite que la brodeuse donnera à cette phrase : «"Thy wife her teeth will grind?" » propose-t-il malicieusement. Ernestina boude, avant de finir la phrase oralement: " "Each time thy watch thou wind, Of love may I thee remind" ». Avec cette association d'idées entre l'amour et la nécessité de remonter le mécanisme d'horlogerie, il est évident que Fowles renvoie son lecteur à Sterne.

Cette scène trouve un écho précis dans Ever After: le père de Matthew Pearce, qui est horloger, a offert à son fils et à sa belle-fille Elizabeth, en cadeau de mariage, une horloge ornée de petits amours, et frappée des initiales des jeunes époux :

On the hinged brass plate at the back, which covers the winding mechanism, is engraved M \& E, 4th April 1845, and above this, the motto, Amor Vincit Omnia. ${ }^{18}$ 
31 Cette description est faite par le narrateur, Bill Unwin, qui se trouve être l'actuel propriétaire de cette horloge transmise de génération en génération à l'occasion des mariages. Bill Unwin cherche à écrire la biographie de son aïeul, à la faire surgir du passé où elle est engloutie, en traitant les carnets intimes comme autant d'ossements à partir desquels, tel un paléontologue, il pourra reconstituer l'être dans sa totalité. Mais ce Matthew auquel il insuffle vie est en partie une fiction, un produit de son imagination. Or, en tant que professeur de littérature britannique le personnage de Bill est censé connaître l'œuvre de Fowles, et l'on voit effectivement que son imagination consciemment ou non - se nourrit à la fois de son expérience personnelle du bonheur conjugal, et de l'image que Fowles donne de la vie quotidienne des victoriens dans ce pastiche de roman du dix-neuvième siècle qu'est The French Lieutenant's Woman. C'est le cas par exemple dans une scène intimiste qui décrit les préparatifs du coucher, les préludes aux ébats amoureux, une scène que Bill «invente» littéralement, car elle n'existe pas dans les carnets de Matthew; Bill la «voit », comme si elle s'étalait sur un écran de cinéma ou sur une scène de théâtre :

I see Matthew tiptoe from the side of a cot. Of two cots... I see him blow out candles; open the little brass plate and wind the clock. He watches Elizabeth at her dressing-table as she loosens her hair. She smiles at his watching smile in the mirror. And he resolves once again-though, by now, perhaps the resolve has become a reflexive, unconscious, continuous thing-not to tell her, to lock up his thoughts. ${ }^{19}$

Dans The French Lieutenant's Woman, Sarah Woodruff, pour séduire Charles, crée une mise en scène : elle fait semblant de s'être foulé la cheville, met ses plus beaux atours, dénoue sa somptueuse chevelure - dont le lecteur sait quel attrait elle exerce sur le héros - et ainsi transformée en damoiselle en détresse, reçoit Charles. Dans le même roman, Ernestina, en promenade avec Charles à Lyme Regis, montre un escalier à son fiancé, en lui rappelant que Jane Austen a mis cet endroit en scène dans Persuasion, quand la jeune Louisa Musgrove fait une mauvaise chute. Dans Ever After, lors de l'épisode de l'ichtyosaure à Lyme Regis, Matthew est tellement choqué qu'il ne s'intéresse pas du tout à la jeune fille qui, au même moment, est tombée sur un sentier étroit au bord de la mer et s'est foulé la cheville. Ces détails, associés à celui d'Elizabeth "loosening her hair » montrent comment s'élabore le palimpseste du roman de Swift, qui combine des allusions à Austen et à Fowles sans jamais explicitement les citer. Matthew Pearce remonte l'horloge gravée tout en pensant à l'amour, et tait les tourments que lui causent le doute ; Charles Smithson plaisante sur la pochette de montre brodée et ment à Ernestina par devoir, pour honorer ses vœux de fiançailles. Si Charles ment par défaut d'amour, Bill désire passionnément que Matthew lui aussi ait menti, mais par amour justement :

No, I don't believe he ever told her about that afternoon in Lyme. [...] Perhaps he meant to tell her. Many times, perhaps, especially in those months before they were married-to get it over with, to exorcize the ghost!-he would have looked for the right moment. (EA 124)

Quand Genette retrace la naissance de la parodie, il cite l'hypothèse de Scaliger selon laquelle, au départ, la parodie serait née de la rhapsodie, et aurait une vocation avant tout comique :

En effet, quand les rhapsodes interrompaient leurs récitations, des amuseurs se présentaient qui retournaient en vue du délassement de l'esprit tout ce qu'on venait d'entendre. Aussi les appela-t-on parodistes, puisque, à côté du sujet sérieux proposé, ils en introduisaient d'autres, comiques. La parodie est donc une rhapsodie retournée, qui par des modifications verbales ramène l'esprit à des objets comiques. 20 
Mais Margaret A. Rose dénonce l'association systématique de la parodie au comique. Selon elle, l'écriture moderniste a réduit la parodie au burlesque, à la farce, et considère ce procédé littéraire avec un certain mépris, alors que les postmodernes ont rendu à la parodie toute sa dimension, à la fois comique et métafictionnelle ${ }^{21}$.

Chez Sterne, l'association entre l'acte d'amour et le mécanisme d'horlogerie est destinée essentiellement à produire un effet comique, burlesque, qui a pour but de tourner en dérision les théories de Locke. Lorsque Fowles associe "watch winding " et amour, sa scène conserve une dimension comique, mais elle est aussi empreinte d'un cynisme amer; quant à Swift, il renverse complètement le processus de la parodie : la référence aux textes antérieurs est bel et bien présente avec l'association entre « clock-winding » et l'atmosphère suggestive, mais aucun effet comique ne subsiste. Le travail intertextuel de Swift transforme ici le travestissement burlesque de Locke par Sterne en un travestissement sérieux de Sterne, via Fowles.

À ce décalage dans les niveaux textuels s'associe le décalage dû au passage du temps et des générations. Le personnage de Bill Unwin, qui possède maintenant l'horloge, ne supporte pas qu'elle ne soit pas remontée, il éprouve une crainte superstitieuse de la voir s'arrêter, et il montre qu'un lien métonymique très fort fait de cette horloge un symbole puissant de son amour disparu :

Ever since that moment of panic, less than two days after her death, when I remembered that the clock had not been wound, (but it had not stopped), it has been my resolution never to let the clock wind down. [...] When I wind the clock, I hold the key which Ruth once held, and holding the key that Ruth once held, I hold the key once held by Matthew. (EA 47)

Il ne subsiste plus la moindre trace de comique ou d'ironie. L'association amour/horloge est récupérée au profit d'un effet pathétique qui a pour but de susciter la compassion du lecteur envers Bill, et elle est également au service de la stratégie globale de l'auteur : le tissage des éléments du roman en un dense réseau regroupant analogies et répétitions obsessionnelles.

On trouve chez Rushdie le même phénomène de retournement, de déviation d'une parodie : il s'agit cette fois d'une parodie d'un passage de Hamlet, dans Tristram Shandy ; en d'autres termes c'est Sterne cette fois-ci qui sert de sas, entre Rushdie et Shakespeare. Dans le roman de Sterne, le père de Tristram Shandy insiste pour que l'accoucheur soit présent quand son fils sera mis au monde, malgré l'obstination de sa femme qui ne veut entendre parler de personne d'autre que la sage-femme. Dès qu'elle entre en couches, le père envoie son serviteur Obadiah, monté sur un puissant cheval d'attelage, chercher le docteur. Celui-ci, à leur insu, se dirige déjà tranquillement vers Shandy Hall, monté sur un petit poney. Hélas pour lui, au détour d'un chemin, la collision est inévitable : il est violemment heurté par le cheval d'Obadiah, galopant ventre à terre, et se retrouve les quatre fers en l'air « in the dirtiest part or a dirty lane, [...] with the broadest part of him sunk about twelve inches deep in the mire $»^{22}$.

Quand le docteur Slop arrive à Shandy Hall pour mettre au monde celui qui - au grand désespoir de son père - sera baptisé Tristram, il se présente donc tout crotté, « unwiped, unappointed, unannealed, with all his stains and blotches on him $»^{23}$, et dans l'esprit du lecteur surgit immédiatement le fantôme du roi Hamlet, qui fut assassiné alors qu'il était « [u]nhousel'd, disappointed, unanel'd,/No reckoning made, but sent to [his] account/ With all [his] imperfections on [his] head $»^{24}$. 
Dans Midnight's Children, le petit Saleem espionne sa mère, coupable de revoir son premier mari en secret; dans le but de lui donner un exemple salutaire, Saleem envoie une lettre anonyme au Commandant Sabarmati pour l'informer des faveurs que sa femme accorde au producteur Homi Catrack. Quand Saleem précise qu'au moment d'expédier son rival dans l'autre monde, le Commandant Sabarmati le trouve «rising from the toilet, his bottom unwiped» (MC 261), il faut lire là une allusion bouffonne à la parodie de Sterne, une surenchère qui transforme le meurtre en farce. En effet, dans cette scène, RushdieSaleem ne fait aucune allusion directe au texte de Hamlet, cependant le participe unwiped, appliqué au postérieur souillé du producteur Catrack, et qui semble appartenir plutôt à un registre scatologique et burlesque, permet de faire planer sur la scène, de façon infiniment discrète, l'ombre de la tragédie shakespearienne, arrivée jusqu'ici en empruntant le sas de la parodie de Sterne.

41 Le procédé utilisé dans Tristram Shandy obéit aux mécanismes du pastiche héroï-comique tels que Genette les répertorie ${ }^{25}$ : le style de l'hypotexte est imité, un nouveau texte noble est appliqué à un sujet vulgaire. Le roi Hamlet est mort " unhousel'd », c'est-à-dire sans avoir reçu l'Eucharistie, il est mort «disappointed", "without having made proper appointment ", sans avoir pu se préparer au rendez-vous suprême avec Dieu, sans s'être confessé ni avoir reçu l'absolution, et il est mort « unanel'd», du verbe « to anele », qui signifie oindre, et surtout donner l'extrême-onction, et qui provient du vieil anglais ele, huile. Sterne transforme «unhousel'd» en «unwiped ", « disappointed» en "unappointed», en jouant sur le sens littéral de "not fitted out, not equipped», et « unanel'd» en « unannealed », qui vient du vieil anglais onaelan, formé sur al, le feu, et dont l'une des définitions est la suivante: «to subject to or undergo some physical treatment, especially heating, that removes internal stress, crystal defects, and dislocations ». Effectivement le docteur Slop, dont le nom se trouve justifié par son accident malencontreux, est extrêmement "stressé", déconfit, trempé, refroidi, bref dans un état avancé de " dislocation ».

Mais si ce passage de Sterne parodie le langage de la pièce de Shakespeare, il en éradique le contenu : dans Hamlet, les mots sont prononcés par le fantôme du Roi, et sont présents à la mémoire de Hamlet lorsqu'il trouve Claudius à ses prières, donc "appointed", tentant de faire sa paix avec son Créateur, ce qui pousse le héros à ne pas tuer son oncle à ce moment-là, parce qu'alors sa vengeance ne serait pas parfaite. Chez Rushdie il ne subsiste qu'une trace à peine discernable du pastiche créé par Sterne; en revanche la charge symbolique est ré-activée, car Catrack, assassiné alors qu'il est encore entaché de toutes ses souillures, ne peut que se présenter directement aux portes de l'enfer. Vu l'importance, dans un contexte musulman, du rite de purification par l'eau (nécessaire avant de pouvoir accéder à la mosquée pour prier), le détail ne prend que plus de relief.

La référence indirecte de Rushdie à Hamlet fait écho à une citation de la même scène $\mathrm{V}$ de l'acte II, citation présente cent pages en amont dans le roman. En effet, lorsque Saleem est confronté, dans la salle de bains, à la perfidie de sa mère, il s'exclame : « - O horrible! my mother $[. ..] »^{26}$, ce qui parodie l'exclamation du Roi Hamlet juste après l'énumération de ses propres péchés ( $\mathrm{O}$ horrible! O horrible! most horrible! ${ }^{27}$ ). Le châtiment infligé à Homi Catrack vise, par procuration, l'inconnu coupable d'avoir posé ses regards sur Amina, la mère de Saleem; en effet, même si Saleem se défend d'avoir voulu pousser le Commandant Sabarmati jusqu'au meurtre, il l'a néanmoins manipulé comme une marionnette, dans le but d'adresser un avertissement solennel à sa mère. 
But Commander Sabarmati was only a puppet; I was the puppet-master, and the nation performed my play-only I hadn't meant it! I didn't think he'd... I only wanted to... a scandal, yes, a scare, a lesson to all unfaithful wives and mothers, but not that, never, no. (MC 262) une mère dont il juge la sexualité embarrassante et inconvenante ; comme Hamlet il considère que sa mère et son beau-père ont une lourde responsabilité dans la mort de son père, et comme Saleem et Hamlet il rêve de vengeance. Bill n'attend pas avant de frapper, et de se venger symboliquement sur son beau-père. Lors de l'épisode de la maquette d'avion brûlée, il cite explicitement les hésitations de Hamlet devant Claudius priant à genoux :

So what should I have done? Drawn my poniard and stabbed his unguarded back?

'Now might I do it pat...'

What I actually did was this... ${ }^{30}$

Si la référence à Hamlet est parfois très directe, il arrive aussi qu'elle soit médiatisée par le sas d'une œuvre tierce, qui n'est autre que le tableau de Millais évoqué plus haut, The Boyhood of Raleigh. Bill, enfant, était comme beaucoup de petits garçons fasciné par les trains, et en particulier par les trains de la Great Western Railway, qu'il allait admirer en se postant près de la voie ferrée. À son insu, il était déjà lié par ce biais à son ancêtre du Devon, Matthew, qui avait rencontré et admiré l'ingénieur Izambard Kingdom Brunel, constructeur de voies ferrées, ponts et autres locomotives. Mais il était aussi métaphoriquement lié à Sir Walter Raleigh : si Raleigh, enfant, se précipitait à la plage 
« to spy the passing ships and sniff the beckoning air », Bill, lui, né loin de la mer, devait se contenter d'espionner les trains, «those galleons of iron and steam, sailing on their way to Oxford, Bristol, and the far south-west " (EA 198).

Sur la même page, Bill décrit Raleigh tel qu'on le voit dans ce qu'il appelle, sans en citer le titre, « Millais's famous painting »:

There he sits, hands clasping his drawn-up velveteen knees, like some child in a Victorian nursery, while a whiskered, brawny mariner, straight out of stage melodrama, flings a histrionic arm towards the horizon. (EA 198)

Comme Rushdie, Swift insiste sur le fantaisiste costume de velours qui brouille les frontières entre l'époque Tudor et l'époque victorienne. Et de même que Rushdie transforme le marin en pêcheur pour l'intégrer à son réseau métaphorique de pêcheurs et de poissons, Swift le récupère en faisant de lui un acteur mélodramatique, qui prend sa place dans la complexe thématique théâtrale du roman. Bill, fasciné par Hamlet et mari d'une actrice, est un narrateur qui théâtralise sans cesse sa narration, avec des personnages qui jouent des rôles, et des situations transformées en véritables scènes, avec dialogues et didascalies; et il cite à plusieurs reprises un célèbre poème de Sir Walter Raleigh qui développe une métaphore théâtrale ${ }^{31}$ pour figurer la vie- c'est-à-dire une métaphore très courante au temps de Shakespeare.

Plus loin dans le roman, Bill compare la vie qu'il a vécue à la destinée qui attendait ce jeune Raleigh :

By my time of life (is that the phrase?) what had the little lad of the sea-shore not achieved? Discovered new lands, founded a colony, won queenly favour, tackled the Spanish Armada. Been soldier, sailor, discoverer, explorer, "Exposing what is mortal and unsure to all that fortune, death, and danger dare..."

Ah, what a thing is man. ${ }^{32}$

Bill, en se comparant à Raleigh, renvoie à Hamlet se comparant au jeune Fortinbras, dont il admire l'audace, l'énergie, la capacité à agir promptement, même pour une cause ridicule, un minuscule lopin de terre, « even for an eggshell »" ${ }^{33}$. Il semble là corroborer le message impérialiste implicite chez Millais. Mais cette citation de l'acte IV est immédiatement suivie par une paraphrase de l'exclamation de Hamlet à l'acte II, « what piece of work is man, how noble in reason [...] », qui se termine par un soupir résigné, " and yet, to me, what is this quintessence of dust? ». Swift rappelle ainsi que l'admiration de Hamlet pour les "hommes d'action" est fugitive, démentie par ses constantes angoisses ontologiques et métaphysiques et par sa conscience de la vanité de toute ambition, de toute entreprise humaine. Quant à Bill, il se sent en fait beaucoup plus proche du Raleigh brisé, enfermé dans la tour de Londres et écrivant « a history of the World. No less. From Adam and Eve until- $»^{34}$ que du Raleigh conquérant célébré par Millais. Si Matthew Pearce est identifié à Raleigh, c'est aussi parce que Raleigh, comme lui, était un explorateur dans le domaine des idées, un intellectuel rongé par le doute, dénonçant les mensonges et les masques de la Cour, refusant le confort des idées reçues. Swift s'approprie ainsi l'espace du tableau, il le restaure et le gratte, nettoie les couches intermédiaires, les détournements, les déviations, pour tenter de mettre à jour une " vérité » qui serait un défi lancé à l'historiographie officielle.

Ce tableau sert de sas interculturel à Rushdie comme à Swift, il sert à mettre en contact les époques et les personnages, à les rendre interchangeables : la vision de Raleigh est filtrée par les poncifs du dix-neuvième siècle, Matthew nous paraît étrangement contemporain dans son rejet passionné de Dieu, et Bill s'engouffre dans le passé, via Fowles ou via 
Millais, pour y trouver refuge, pour tenter d'oublier le vide béant que la mort de sa femme a ouvert dans sa vie. Il n'y parvient pas, et lorsqu'il place la vie de Matthew et d'Elizabeth Pearce sous un microscope, c'est en fait le couple qu'il formait avec Ruth qu'il remet en question. Les analogies entre les personnages ne sont pas « originées » : tantôt c'est Matthew qui est identifié à Raleigh, ce qui, par syllogisme, identifie Bill à Raleigh, tantôt c'est Bill qui est identifié à Raleigh, ce qui crée une analogie entre Raleigh et Matthew. On retrouve ici un des aspects caractéristiques du postmodernisme tels que Brian McHale les définit: le débordement hors des cadres, le brouillage des limites ontologiques, le glissement d'une époque dans une autre ou d'un espace dans un autre. Mais le «sas » du tableau permet de rester au niveau métaphorique ou symbolique, car chez Swift jamais ces brouillages ne sont surréalistes ou " magiques », comme ils peuvent l'être chez Rushdie ou dans la littérature latino-américaine.

Lorsque Bill décrit le paysage qu'il voyait, enfant, près de la voie ferrée, il insiste sur le fait qu'il avait sous les yeux, simultanément, des êtres, des objets et des moyens de communication appartenant à une multitude de périodes différentes; il compare le paysage à « those contrived scenes in a children's encyclopaedia, depicting the theme of 'Old and New'" (EA 199), et le qualifie de "living palimpsest». Cette scène qui semble sortie d'un livre est une parfaite illustration du roman que Swift a écrit, et s'il la décrit ce n'est pas fortuit, mais bien pour offrir une métaphore auto-réflexive de sa thématique et de sa méthode : il écrit un livre qui est lui-même un palimpseste, dans la mesure où il superpose en couches successives les références intertextuelles, mais en les synchronisant, en créant des personnages qui sont des avatars interchangeables les uns des autres ; « the people go, the patterns remain » (EA 47).

Saleem et Bill se donnent tous les deux le même modèle, celui de Hamlet, et défendent avec plus ou moins de ferveur l'honneur menacé de leur père. Néanmoins, et très ironiquement, il s'avère qu'aucun des deux n'est le fils de son père. Confrontés aussi brutalement l'un que l'autre à la révélation cruelle de leur bâtardise, ils se retrouvent soudain dans l'obligation de renoncer à une identité qu'ils avaient patiemment élaborée et consolidée ; leur être, vide de toute substance, est alors voué à la désintégration pour Saleem, au suicide pour Bill. Cette rupture de la filiation métaphorise la dérive et l'errance de l'écriture contemporaine, privée d'origine, privée d'autorité auctoriale, privée de vérité et d'idéologie. L'identification de Saleem à l'Inde et de Bill/Matthew à Raleigh, et donc à la grandeur de l'Angleterre, transforme cette quête du père et de leur histoire individuelle en quête impossible de l'Histoire collective, de la vérité historique, et même de toute vérité.

Les romans modernistes célébraient l'épiphanie, la révélation fulgurante, illuminatrice, qui permettait d'appréhender les choses dans leur globalité. Virginia Woolf conseillait à un poète d'observer le monde en laissant libre cours à son sens du rythme, « until the taxis are dancing with the daffodils, until a whole has been made from all these separate fragments $\aleph^{35}$. Dans les romans postmodernistes, l'appréhension globale, la compréhension et la connaissance parfaites sont impossibles; tous les fragments ne peuvent être assemblés. Dans Grimus, Rushdie a inventé un personnage qui fabrique des puzzles et s'amuse à en subtiliser un morceau, pour qu'ils ne puissent jamais être complétés; dans une nouvelle de Robert Nye, "The Facts of Life", le narrateur se rappelle avoir commencé un puzzle sur lequel était reproduit - encore ! - notre tableau de Millais ( the Boyhood of Raleigh in one thousand two hundred and fifty pieces ${ }^{36}$ ), pour s'apercevoir, alors qu'il l'avait presque achevé, qu'il lui manquait un morceau, 
précisément l'index du marin. Dans la nouvelle cet index manquant symbolise l'incapacité du père à expliquer à son fils " the facts of life ", mais surtout le sens de la vie, présentée comme une sinistre comédie consacrée à la guerre. Ce père n'a pas connu son propre père, mort au front pendant la première guerre mondiale, et cette absence ou cette ineptitude des pères, de nouveau, métaphorise le sentiment postmoderniste que rien « n'autorise » l'écriture, et qu'aucun sens ne sera désigné au lecteur, qui devra seul, comme le dit Barthes, non pas « déchiffrer " mais " démêler » le texte, "fait d'écritures multiples, issues de plusieurs cultures et qui entrent les unes avec les autres en dialogue, en parodie, en contestation ${ }^{37}$.

La fonction des «sas" est donc plurielle: ils permettent de remettre en question l'historiographie de rigueur, tout en soulignant la nécessité de mêler les cultures en une chimère hybride, par définition, mais harmonieuse. Les sas permettent aussi de remonter une filiation littéraire vers sa source, et problématisent la relation entre réalité et représentation. Si ces deux romans renvoient aux mêmes œuvres, c'est une coïncidence, mais c'est également symptomatique de ressemblances plus profondes : il y a un souci constant, chez Swift comme chez Rushdie, de créer des romans où fiction et métafiction sont inextricablement mêlées, et figurées par les mêmes métaphores, ce qui fait que l'une renvoie à l'autre dans un rapport inoriginé. Swift et Rushdie savent créer ces romans hybrides, tendus d'une part entre cette attitude de «solipsistic navel-gazing and empty ludic game playing » qu'évoque Linda Hutcheon ${ }^{38}$, et d'autre part la volonté (assortie de précautions et d'ironie) de représenter une réalité, de chercher une vérité, et de s'engager politiquement. Ils savent, tout en déconstruisant constamment leur fiction, susciter néanmoins chez les lecteurs l'adhésion à une histoire et satisfaire leur désir, jugé démodé par les critiques, mais toujours aussi vif chez les lecteurs "non professionnels", de s'identifier à des personnages humains et humanistes. Bref, Rushdie comme Swift se servent avec brio des expérimentations modernistes et de leurs prolongements postmodernistes.

Walton Litz suggérait avec un certain dédain, en 1986, qu'avec la littérature postmoderniste, dont l'appellation évoque, dit-il, «post-mortem or post-coital », «the fun is over $"$ ". Avec des romanciers comme Rushdie et Swift, il semblerait plutôt que la fête continue.

\section{BIBLIOGRAPHIE}

Barthes, Roland. Le Bruissement de la langue. Paris : Éditions du Seuil, coll. « Points », 1984.

Eco, Umberto. Reflections on The Name of the Rose. Trad. W. Weaver. London: Secker \& Warburg, 1985.

Fitzgerald, F.S. Tender is the Night (1934). Harmondsworth: Penguin, 1998.

Fowles, John. The French Lieutenant's Woman (1969). London: Pan Books, 1987.

Genette, Gérard. Figures III. Paris : Éditions du Seuil, 1972. 
Genette, Gérard. Palimpsestes. Paris : Éditions du Seuil, 1982.

McHale, Brian. Postmodernist Fiction. London: Methuen, 1987.

Millais, J.G. The Life and Letters of Sir John Everett Millais. London: Methuen, 1899.

Rose, Margarer A. Parody: Ancient, Modern, and Post-Modern. Cambridge: Cambridge UP, 1993.

Rushdie, Salman. Imaginary Homelands. London: Granta Books, 1991.

Rushdie, Salman. Midnight's Children (1981). London: Pan Books (Picador), 1982.

Rushdie, Salman. Shame (1983). London: Pan books (Picador), 1984.

Said, Edward. Orientalism. New York: Random House, 1978.

Scott, Paul. The Jewel in the Crown (1966). London: Pan Books.

Sterne, Lawrence. Tristram Shandy (1759-1767). Harmondsworth: Penguin, 1967.

\section{NOTES}

1. Umberto Eco, «Postmodern, Irony, the Enjoyable ", in Reflections on The Name of the Rose, traduit par W. Weaver. London: Secker \& Warburg, 1985.

2. Stevens, cité par John Guille Millais in The Life and Letters of Sir John Everett Millais, London: Methuen, 1899, vol. II, 17.

3. Dans l'essai « Outside the Whale », in Imaginary Homelands, London: Granta Books, 1991.

4. Paul Scott, The Jewel in the Crown (1966). London: Pan Books, 34.

5. Francis Scott Fitzgerald évoque «Mrs Burnett's vicious tracts» (Tender is the Night, 43) et l'on trouve dans le même roman (80) la collocation «vicious sentimentality », qui s'applique bien à l'effet que veut produire Rushdie.

6. S. Rushdie, «In Good Faith » in IH, 394. (La citation vient de MC 38.)

7. Roland Barthes, Le Bruissement de la langue, Paris : Éditions du Seuil, coll. « Points ", 1984, 88.

8. MC 136, c'est moi qui souligne.

9. Voir Genette, Figures III, 233-234.

10. " you better get a move on or you'll die before you get yourself born », MC 38 .

11. Lawrence Sterne, Tristram Shandy, Harmondsworth: Penguin, 1967, 35.

12. Edward Said, Orientalism, New York: Random House, 1978.

13. S. Rushdie The Satanic Verses (1988), New York: Viking Penguin, 406.

14. «In Good Faith », in Imaginary Homelands, op. cit., 394.

15. Ibid.

16. Brian McHale, Postmodernist Fiction, London: Methuen, 1987, chapitre 7.

17. John Fowles, The French Lieutenant's Woman (1969). London: Pan Books, 1987, 291.

18. Graham Swift, Ever After. London: Pan Books, 1992, 46.

19. EA 124 , je souligne.

20. Scaliger cité par G. Genette, in Palimpsestes, op. cit., 21.

21. Voir M. A. Rose, Parody: Ancient, Modem, and Post-Modern. Cambridge: Cambridge UP, 1993.

22. Lawrence Sterne, Tristram Shandy, op. cit., II, x, 124 et 125.

23. Lawrence Sterne, Tristram Shandy, op. cit., II, x, 126, souligné par l'auteur.

24. William Shakespeare, Hamlet, I, v, 77-79.

25. Gérard Genette, Palimpsestes, op. cit., 29-30.

26. MC 161.

27. Hamlet, I, v, 80.

28. Hamlet, III, iv, 93. 
29. Ibid., III, iv, 81.

30. EA 64 ; la citation de Hamlet vient de III, NI, 73.

31. "Our Mothers' wombes the tiring houses be/Where we are dressed for this short Comedy... » (cité dans EA 233).

32. EA 232, cf. Hamlet IV, iv, 51-52, et II, ii, 303.

33. Hamlet, IV, iv, 53.

34. EA. 232.

35. V. Woolf, « Letter to a Young Poet », Collected Essays, 2: 185, cité par François Gallix, Le Roman britannique du vingtième siècle, Paris: Masson, 1995.

36. Robert Nye, « The Facts of Life ", in The Facts of Life and Other Fiction, London: Sheil Land, 1983.

37. Roland Barthes, «La mort de l'auteur », dans Le Bruissement de la langue, op. cit. 69.

38. Linda Hutcheon utilise cette formule pour résumer l'hostilité de certains critiques envers les romans postmodernistes, citée dans E. Smyth, Postmodernism and Contemporary Fiction, London: Batsford, 1991

39. Walton Litz, "Modernist Making and Self-Making », TLS 18 oct. 1986, cité par Smyth, Postmodernism and Contemporary Fiction, op. cit., 35.

INDEX

oeuvrecitee Midnight's Children, Satanic Verses (The), Ever After 